\title{
Natural hybridization and asymmetric introgression at the distribution margin of two Buddleja species with a large overlap
}

Rong-Li Liao ${ }^{1,2 \dagger}$, Yong-Peng Ma ${ }^{1 \dagger}$, Wei-Chang Gong ${ }^{3}$, Gao Chen ${ }^{1}$, Wei-Bang Sun ${ }^{1 *}$, Ren-Chao Zhou ${ }^{4^{*}}$ and Tobias Marczewski ${ }^{1}$

\begin{abstract}
Background: Natural hybridization in plants is universal and plays an important role in evolution. Based on morphology it has been presumed that hybridization occurred in the genus Buddleja, though genetic studies confirming this assumption have not been conducted to date. The two species B. crispa and B. officinalis overlap in their distributions over a wide range in South-West China, and we aimed to provide genetic evidence for ongoing hybridization in this study.

Results: We investigated the occurrence of hybrids between the two species at the southern-most edge of the distribution of B. crispa using five nuclear loci and pollination experiments. The genetic data suggest substantial differentiation between the two species as species-specific alleles are separated by at least 7-28 mutations. The natural hybrids found were nearly all F1s (21 of 23), but backcrosses were detected, and some individuals, morphologically indistinguishable from the parental species, showed introgression. Pollen viability test shows that the percentage of viable pollen grains was $50 \pm 4 \%$ for $B$. crispa, and $81 \pm 2 \%$ for $B$. officinalis. This difference is highly significant $(t=7.382$, $p<0.0001$ ). Hand cross-pollination experiments showed that $B$. crispa is not successful as pollen-parent, but $B$. officinalis is able to pollinate $B$. crispa to produce viable hybrid seed. Inter-specific seed-set is low (8 seeds per fruit, as opposed to about 65 for intra-specific pollinations), suggesting post-zygotic reproductive barriers. In addition, one of the reference populations also suggests a history of introgression at other localities.
\end{abstract}

Conclusions: The occurrence of morphologically intermediate individuals between B. crispa and B. officinalis at Xishan Mountain is unequivocally linked to hybridization and almost all examined individuals of the putative hybrids were likely F1s. Despite pollination experiments indicating higher chances for introgression into B. officinalis (hybrids only produced viable seed when crossed with $B$. officinalis), observed introgression was asymmetrical into $B$. crispa. This could be due to seeds produced by hybrids not contributing to seedlings, or other factors favoring the establishment of backcrosses towards B. crispa. However, further research will be needed to confirm these observations, as the small number of plants used for the pollination experiments could have introduced an artifact, for example if used individuals were more or less compatible than the species average, and also the small number of loci used could convey a picture of introgression that is not representative for the whole genome.

Keywords: Asymmetric introgression, Buddleja, Hybridization, Nuclear genes, Reproductive isolation

\footnotetext{
*Correspondence: wbsun@mail.kib.ac.cn; zhrench@mail.sysu.edu.cn

${ }^{\dagger}$ Equal contributors

'Kunming Botanical Garden; Key Laboratory for Plant Diversity and

Biogeography of East Asia, Kunming Institute of Botany, Chinese Academy of

Sciences, Kunming 650201, Yunnan, China

${ }^{4}$ State Key Laboratory of Biocontrol and Guangdong Provincial Key

Laboratory of Plant Resources, Sun Yat-sen University, Guangzhou 510275,

China

Full list of author information is available at the end of the article
}

\section{Biomed Central}

(c) 2015 Liao et al. This is an Open Access article distributed under the terms of the Creative Commons Attribution License (http://creativecommons.org/licenses/by/4.0), which permits unrestricted use, distribution, and reproduction in any medium, provided the original work is properly credited. The Creative Commons Public Domain Dedication waiver (http:// creativecommons.org/publicdomain/zero/1.0/) applies to the data made available in this article, unless otherwise stated. 


\section{Background}

Natural hybridization is ubiquitous in plants and has several evolutionary consequences including the origin and/or transfer of genetic adaptations, the origin of new ecotypes or species, and the reinforcement or breakdown of reproductive barriers [1-5]. For closely related species with sympatric distribution, the formation and maintenance of reproductive isolating barriers is an important issue in speciation [6-8]. In such cases, species boundaries could be maintained by the elimination of intermediate hybrids due to low $F_{1}$ fertility or hybrid breakdown $[7,9]$, or by $\mathrm{F}_{1}$ dominated hybrid zones, in which $\mathrm{F}_{1} \mathrm{~s}$ exhibit apparent habitatmediated superiority over other hybrid classes [10]. The latter is an extreme case scenario, and even a small number of hybrids beyond the F1 generation might be enough to provide a genetic bridge enabling introgression [10,11].

Buddleja crispa Benth. and B. officinalis Maxim are two species in the family Scrophulariaceae, both having the habit of shrubs or, in the case of $B$. officinalis, rarely small trees, reaching a height of about $3 \mathrm{~m}$. B. crispa grows mostly at altitudes of above $2000 \mathrm{~m}$, and prefers exposed rocky habitats and dry river valleys [12]; this species has a distribution ranging from Afghanistan into the eastern Himalayas, where it reaches into the higher parts of the Himalayan foothills of South-West China in Yunnan and Sichuan. The distribution of B. officinalis is restricted to comparably lower altitudes in South to South-west China, but it has a large overlap with $B$. crispa in the foothills, where both altitudinal ranges meet at an approximate altitude of $1500-2500 \mathrm{~m}$; $B$. officinalis prefers forest edges on mountains, and thickets on riverbanks. Both species are predominantly outcrossing, though partial self-fertility and autogamy were occasionally observed in B. crispa [13]. Both species are diploid with chromosome numbers $2 n=38$, and have an intra-specifically variable morphology, including flower color, leaf size and shape, and indumentum thickness. Furthermore, both species flower in early spring (B. crispa - March to April; B. officinalis - February to May [14]; and are likely to share pollinators, mostly butterflies [15-17]; however, despite the extensive overlap, no hybrids between the species have been reported to date. When the two species grow sympatrically, B. crispa is mostly found at the higher altitudes, often growing under extreme conditions on sheer rock faces, mostly with very little soil available, and very exposed. In this habitat the plants frequently remain rather small, not exceeding $1 \mathrm{~m}$ in height. $B$. officinalis is never found growing under these extreme conditions, and mostly replaces $B$. crispa at lower altitudes, where it is often growing amongst other vegetation below the canopy of larger trees, or on disturbed ground, but always with a considerable layer of soil available. Hence the two species seem to have different ecological requirements in sympatry, but $B$. crispa does grow in B. officinalis habitat, when the species is not present, and at sympatric sites the species are in close contact due to the proximity of respectively suitable sites (e.g. exposed rocks and cliffs amidst a forest covered slope). Although no hybrids between the species have been reported, numerous intermediate individuals have been discovered at the south-eastern most range limit of B. crispa, namely Xishan Mountain near Kunming city in Yunnan (Sun Weibang, personal observation).

Geological records suggest profound climatic changes in the region over the last 30,000 years leading to three major changes in floral composition, with the last having occurred 13,000 years ago [18]. Additionally, temperature changes led to significant forest range changes, and changes in species abundance up to about 2500 to 1500 years ago [19]. It is likely, that these environmental changes will also have resulted in range contraction and expansion of B. crispa and B. officinalis. The geographical location of the potential hybrid zone is especially interesting, as it allows investigating questions about reproductive isolation between species at the extremes of their distribution range. In the present study, our main aims are to test the hybridization hypothesis, and to investigate reproductive isolation barriers in this special location at the distribution margin of one of the two species. The specific questions we want to ask are: (1) Are these morphologically intermediate individuals really hybrids between $B$. crispa and B. officinalis? (2) What is the composition of the hybrid zone on Xishan Mountain? Is there any introgression between the two species? and (3) Is there any evidence for reproductive barriers between these two species?

\section{Results}

Morphological analysis for B. officinalis, B. crispa and their putative hybrid

At all sampling sites, B. officinalis and B. crispa could easily be distinguished using the four morphological characters: leaf shape, leaf margin, leaf base including petiole, and indumentum on the adaxial leaf surface (Fig. 1). Putative hybrid individuals had morphological characters intermediate between $B$. crispa and B. officinalis, although leaves are often distinct in shape from both of the parents, as the widest part of the leaf lamina is more central, and hence conforming to an elliptic shape. The Welch statistic calculated for the three Buddleja taxa we examined indicated significant differences for the leaf width $(F=51.236, P<0.001)$. The leaf width of $B$. officinalis was smaller than that of both $B$. crispa and the putative hybrids $(P<0.001$ for each 


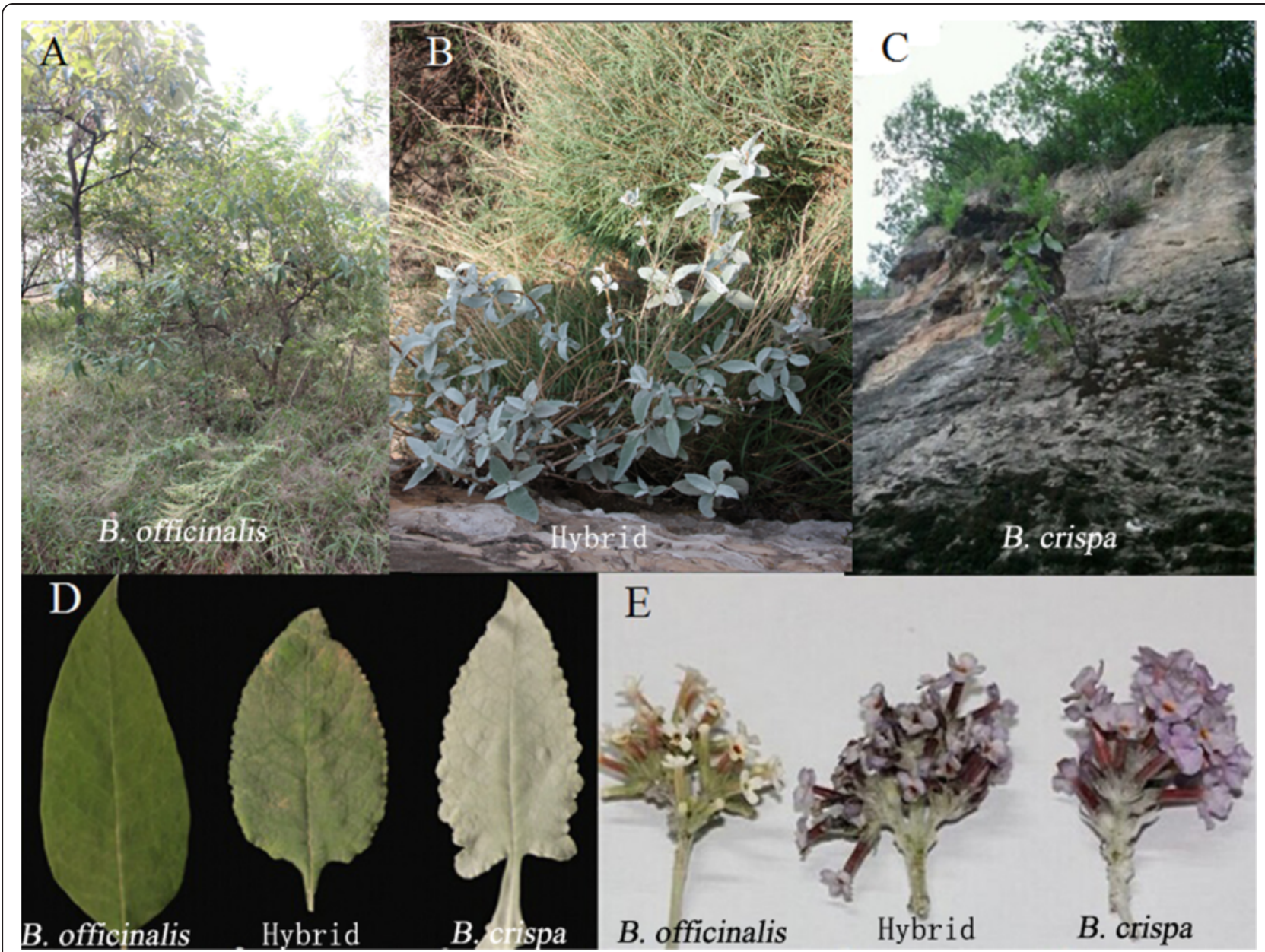

Fig. 1 Habitats $(\mathbf{a}, \mathbf{b}, \mathbf{c})$, leaf (d) and flower characteristics (e) of B. officinalis, B. crispa and the putative hybrids

comparison); however, there was no significant difference between $B$. crispa and the putative hybrid $(P=0.312)$ (Table 1).

While the leaf length was not significantly different between the three taxa $(F=0.563, P=0.572$, Table 1$)$, ratios of leaf length to leaf width were significantly different between all three taxa $(F=52.207, P<0.001)$. B. officinalis and B. crispa had the greatest and smallest ratios of leaf length to leaf width, respectively, with the putative hybrid being intermediate (Table 1).

Table 1 Morphological traits used to distinguish B. officinalis, B. crispa and their putative hybrid

\begin{tabular}{|c|c|c|c|c|c|c|}
\hline Character & B. officinalis & B. crispa & Putative hybrid & $\mathrm{F}$ & Welch & $P$ value \\
\hline Leaf shape & Narrowly ovate & Ovate & Ovate-elliptic & & & \\
\hline Leaf margin & Entire & Crenate & Sinuate & & & \\
\hline Adaxial leaf surface indumentum & Glabrescent & Densely tomentose & Puberulent & & & \\
\hline Leaf base including petiole & Cuneate, free & Auriculate, winged & Cuneate to decurrent & & & \\
\hline Corolla color & Yellow-white & Lilac to purple & Lilac & & & \\
\hline$L(\mathrm{~cm})$ & $13.35 \pm 1.69$ & $13.54 \pm 2.41$ & $13.89 \pm 1.90$ & 0.563 & & 0.572 \\
\hline$W(\mathrm{~cm})$ & $4.99 \pm 0.73^{\mathrm{a}}$ & $7.35 \pm 1.36^{b}$ & $6.85 \pm 1.07^{b}$ & & 51.236 & $<0.001$ \\
\hline$L / W$ & $2.70 \pm 0.36^{\mathrm{a}}$ & $1.86 \pm 0.30^{\mathrm{b}}$ & $2.04 \pm 0.14^{c}$ & & 52.207 & $<0.001$ \\
\hline
\end{tabular}

mean \pm standard deviation are shown for the three traits

$L$ leaf length, $W$ leaf width, $L / W$ ratio of leaf length to leaf width

$\mathrm{a}, \mathrm{b}, \mathrm{c}$ :the means with different superscripts are significantly different from each other at the 0.05 level and based on Tamhane's $\mathrm{T} 2$ test 


\section{Pollen viability test and hand pollination experiments} In total 714 pollen grains of $B$. crispa and 445 pollen grains of $B$. officinalis were examined for viability. Based on staining with MTT, the percentage of viable pollen grains in just dehisced anthers was $50 \pm 4 \%$ for $B$. crispa, and $81 \pm 2 \%$ for $B$. officinalis. This difference is highly significant $(t=7.382, p<0.0001)$.

Cross-pollination treatments showed a wide range of fruit set, ranging from $6 \%$ (B. officinalis $9 \times$ B. crispa $\left.{ }^{\top}\right)$ to $84 \%$ (putative hybrid $+\times B$. officinalis ${ }^{\lambda}$ ) (Table 2). However, the number of seeds per fruit was one order of magnitude higher for the intraspecific crosses $B$. officinalis $(q) \times B$. officinalis $\left(\sigma^{\top}\right)$ and B. crispa $(+) \times B$. crispa (ठ) ( $\sim 65$ compared to $0.43-8$, Table 2$)$, resulting in many more viable seeds for these crosses, even with lower fruit set. In interspecific crosses there was a marked difference between the two species with regards to success as pollen donor and pollen recipient. While crosses with $B$. crispa as maternal parent always produced at least some viable seed, no viable seeds were produced in any heterospecific cross with $B$. crispa as paternal parent. $B$. officinalis on the other hand was always a successful pollen donor (Table 2).

\section{Sequence analysis of four low-copy nuclear genes and nrETS}

GapC1

The alignment of gapC1 spanned $608 \mathrm{bp}$, including only one 1-bp indel, which distinguished $B$. officinalis alleles from $B$. crispa alleles. Haplotype network analysis identified two highly divergent clusters separated by $28 \mathrm{nu}$ cleotide substitutions (Fig. 2a). Only one haplotype was present in B. officinalis individuals, which represents one of the clusters, while haplotypes present in B. crispa generally conform to the other; the only exception to this pattern are three individuals of B. crispa (Z13, Z15 and Z22) which had the haplotype found in B. officinalis. With regards to the putative hybrid individuals, all but two (P18 and P20) had two divergent haplotypes, each originating from one of the diverged clusters. The two individuals (P18 and P20) were homozygous at this locus, with P18 having the same sequence as B. officinalis and P20 possessing a unique haplotype nested within the B. crispa cluster.

\section{GapC2}

The alignment of gapC2 spanned 596 bp including one 1-bp indel distinguishing $B$. officinalis alleles from $B$. crispa alleles. Haplotype network analysis identified two major clusters separated by 7 nucleotide substitutions (Fig. 2b). One cluster comprised 4 out of 5 haplotypes of $B$. officinalis, the other8 out of 10 haplotypes of $B$. crispa. Additionally, two haplotypes from seven individuals of B. crispa (Z6, Z13, Z14, Z15, Z17, Z18 and Z22) were nested within the $B$. officinalis cluster, and one haplotype from one individual of B. officinalis (M2) was nested within the $B$. crispa cluster.

Of the putative hybrid individuals, all but three (P13, P18 and P20) had two divergent haplotypes, one nested within each of the two divergent clusters. Two individuals (P18 and P20) were homozygous at this locus, having haplotypes from the $B$. officinalis and B. crispa clusters, respectively. Individual P13 had two haplotypes found in the B. crispa cluster.

\section{DefA}

The length of the sequenced fragment of $\operatorname{def} A$ was 349 bp for all individuals. Haplotype network analysis identified two clusters separated by four nucleotide substitutions (Fig. 2c). All 9 haplotypes of B. officinalis belonged to one of these clusters, while the other cluster contained 4 of the 5 haplotypes found in B. crispa. The remaining haplotype found in one individual of $B$. crispa (Z22) was identical to the major haplotype of B. officinalis. Of the putative hybrid individuals all but one (P18) had two divergent haplotypes, one from each of the two

Table 2 Fruit set, seed number, seed viability and number of viable seeds per fruit for nine pollination combinations among $B$. officinalis, B. crispa and their putative hybrid

\begin{tabular}{|c|c|c|c|c|c|c|c|}
\hline $\begin{array}{l}\text { Pollen recipient } \\
\text { (9) }\end{array}$ & $\begin{array}{l}\text { Pollen donor } \\
\left(\partial^{3}\right)\end{array}$ & $\begin{array}{l}\text { Number of } \\
\text { flowers }\end{array}$ & $\begin{array}{l}\text { Number of } \\
\text { fruits }\end{array}$ & $\begin{array}{l}\text { Fruit set } \\
(\%)\end{array}$ & $\begin{array}{l}\text { Mean number of seeds } \\
\text { per fruit }\end{array}$ & $\begin{array}{l}\text { Seed viability } \\
(\%)\end{array}$ & $\begin{array}{l}\text { Mean number of viable } \\
\text { seeds per fruit }\end{array}$ \\
\hline \multirow[t]{3}{*}{ B. crispa } & B. crispa & 20 & 11 & 55 & 65.50 & 34 & 22.27 \\
\hline & B. officinalis & 24 & 14 & 58 & 8.00 & 68 & 5.44 \\
\hline & Putative hybrid & 27 & 15 & 55 & 2.20 & 70 & 1.54 \\
\hline \multirow[t]{3}{*}{ B. officinalis } & B. crispa & 32 & 2 & 6 & 1.00 & 0 & 0.00 \\
\hline & B. officinalis & 23 & 10 & 43 & 65.00 & 91 & 59.15 \\
\hline & Putative hybrid & 37 & 18 & 48 & 1.94 & 57 & 1.11 \\
\hline \multirow[t]{3}{*}{ Putative hybrid } & B. crispa & 31 & 19 & 61 & 2.79 & 0 & 0.00 \\
\hline & B. officinalis & 38 & 32 & 84 & 4.63 & 53 & 2.45 \\
\hline & Putative hybrid & 45 & 7 & 15 & 0.43 & 0 & 0.00 \\
\hline
\end{tabular}

aviable seeds per fruit $=$ mean seed number $\times$ seed viablility 


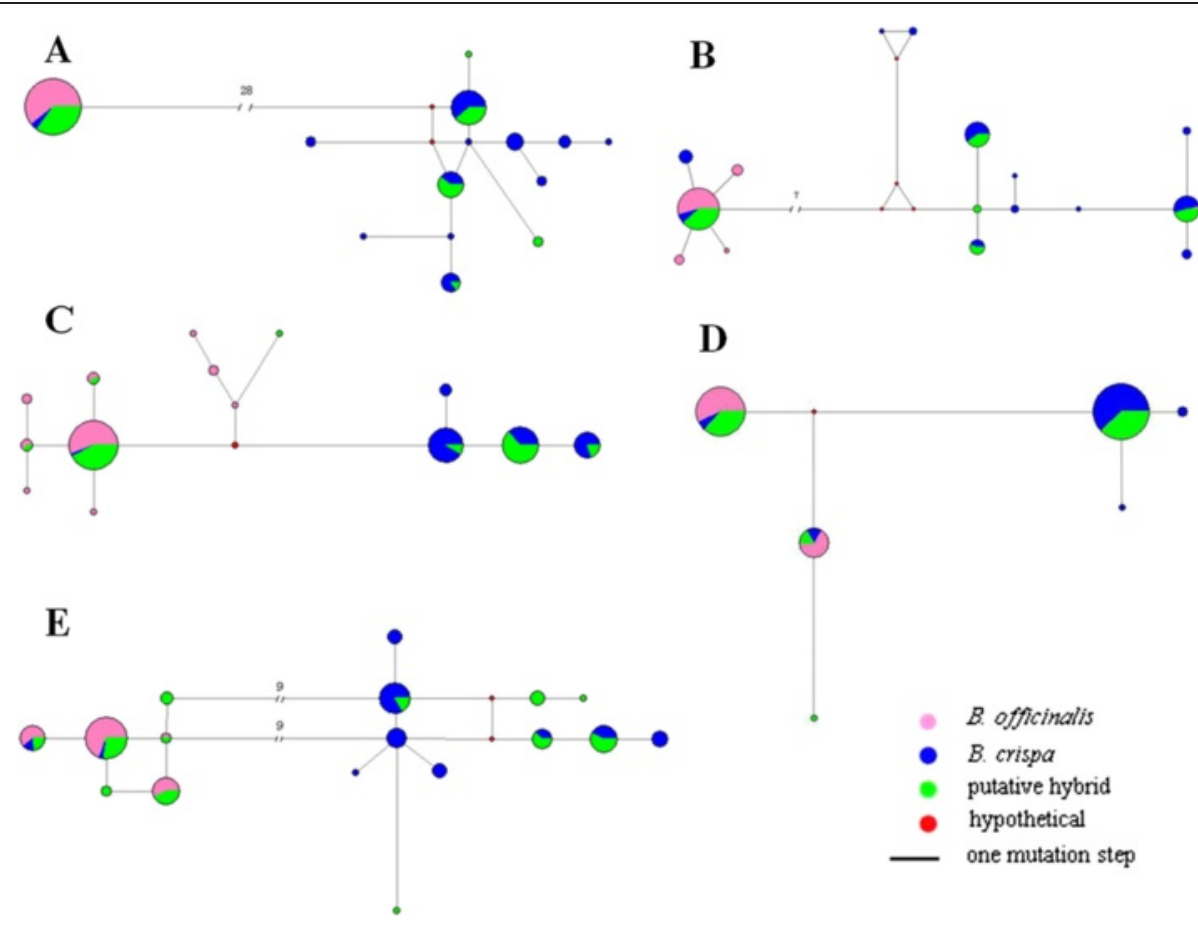

Fig. 2 Haplotype networks for gapC1 (a), gapC2 (b), defA (c), fl1 (d) and nrETS (e). Pink, blue and green circles represent haplotypes of B. officinalis, B. crispa and their putative hybrid. Small red circles represent hypothetical or unsampled haplotypes. The number of mutations separating two haplotypes is indicated by the length of the connecting lines unless the number was shown on the line

clusters. Individual P18 was homozygous at this locus for a B. officinalis haplotype.

\section{FI1}

The alignment of $f l 1$ spanned 270 bp including one 3-bp indel distinguishing B. officinalis and B. crispa alleles. As in the cases above the two clusters identified employing haplotype network analysis corresponded largely to haplotypes found in B. officinalis and B. crispa, respectively. The clusters were separated by 6 nucleotide substitutions (Fig. 2d), and B. officinalis haplotypes grouped exclusively in one cluster. While 3 of 5 haplotypes of $B$. crispa were in the other cluster, 2 haplotypes from six individuals of B. crispa (Z9, Z13, Z15, Z20, Z21 and Z22) clustered with B. officinalis haplotypes. With the exception of P20, all of the putative hybrid individuals had two divergent haplotypes, one from each of the two clusters; individual P20 was homozygous for a B. crispa haplotype at this locus.

\section{NrETS}

The length of the sequenced fragment of the nrETS region was 337 bp in all individuals. Haplotype network analysis identified two clusters separated by 9 nucleotide substitutions (Fig. 2e). All four haplotypes of B. officinalis were grouped in one cluster, and 8 of 10 haplotypes of $B$. crispa were grouped in the other cluster. Interestingly, 2 haplotypes from three individuals of B. crispa (Z13, Z15 and Z22) clustered with $B$. officinalis. The putative hybrid individuals showed the same pattern as before, all but one individual (P18) having two divergent haplotypes, one from each of the two clusters; individual P18 was homozygous for a B. officinalis haplotype.

\section{NewHybrids analysis}

Posterior probabilities for the assignment of individuals to certain genotype classes (parent, F1, F2, backcross) were obtained with the program NewHybrids. Individuals previously identified as B. officinalis based on morphological characters, were all assigned to $B$. officinalis with high posterior probabilities $(>0.977)$. Of the 24 individuals morphologically identified as $B$. crispa, 20 individuals were assigned to $B$. crispa with high posterior probabilities $(>0.982)$, but 3 individuals were assigned to the F1 class (Fig. 3a - Z13, Z15 and Z22) and one to B. crispa with much lower probability (Z6). Of the 23 individuals morphologically identified as putative hybrids, 21 individuals were assigned to the $F_{1}$ class with high posterior probabilities ( $>0.969$ ); two individuals, however, were classed as B. officinalis (P18, 0.949) and B. crispa (P20, 0.991), respectively (Fig. 3a; Additional file 1: Table S1).

\section{Structure analysis}

The most likely number of clusters (K) for the whole dataset, as determined by the highest $\Delta \mathrm{K}$ [20], was 


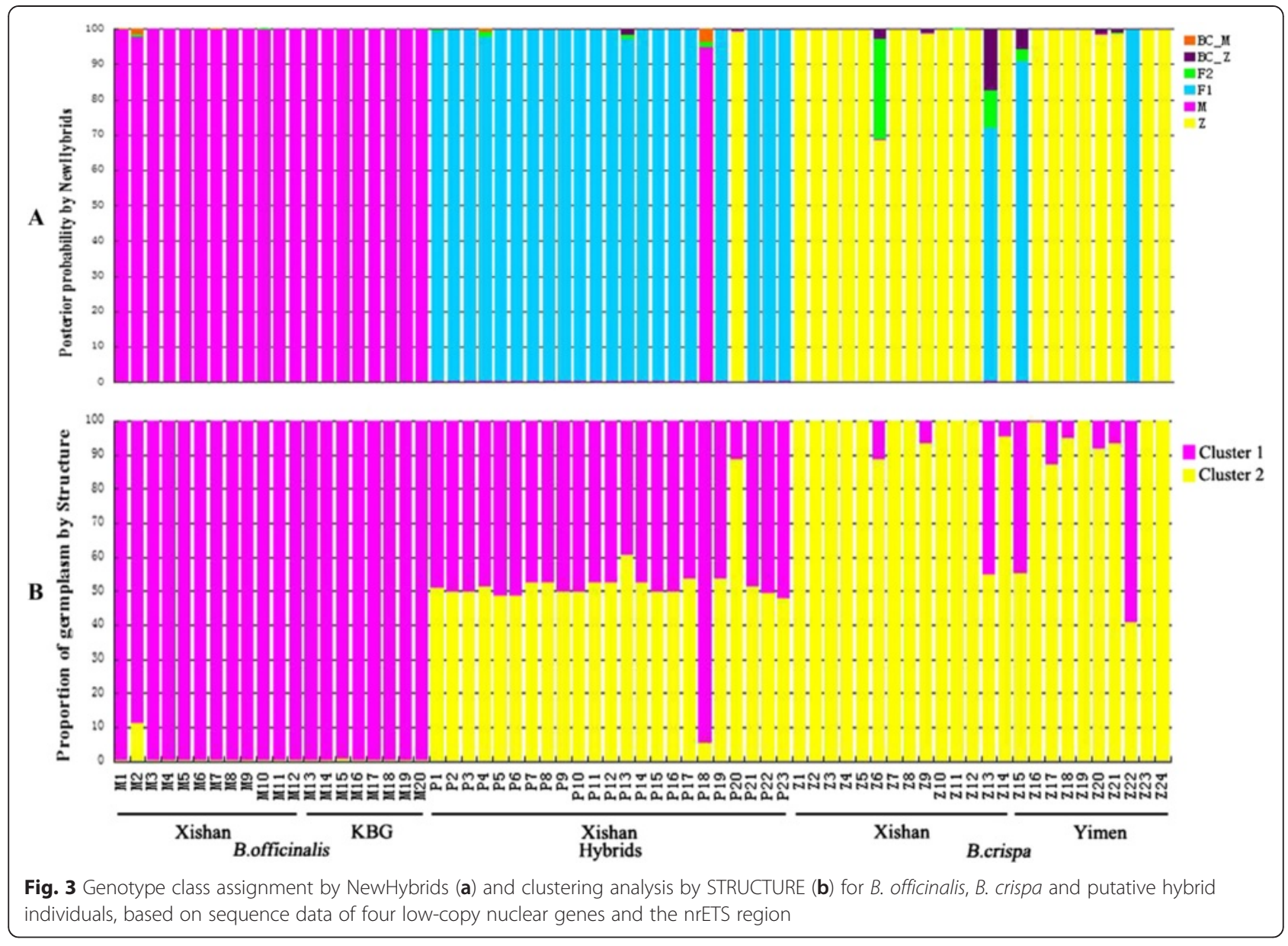

chosen as the true value of $\mathrm{K}$. The Structure analysis for the three taxa yielded a highest $\Delta \mathrm{K}$ value for $\mathrm{K}=2$ (Additional file 2: Figure S1), indicating that two genetic clusters were sufficient to explain structures observed in the three groups. When $\mathrm{K}=2$, alleles of individuals morphologically identified as $B$. officinalis mostly originate from one cluster $(\mathrm{q}=0.991 \pm 0.024)$, whereas alleles from 21 of 24 individuals morphologically identified as $B$. crispa originate mostly from the other $(\mathrm{q}=0.910 \pm 0.168)$. Therefore, the two clusters were interpreted as corresponding to B. officinalis and B. crispa, respectively. The same three individuals with $B$. crispa morphology that were classed as F1s by NewHybrids had alleles derived from both clusters in about equal proportion (Fig. 3b; Additional file 1: Table S2, Z13, Z15 and Z22; q_cluster $2=0.548,0.550$ and 0.406 , respectively).

Nearly all of the putative hybrid individuals, 21 out of 23, had alleles in equal proportion from both clusters (q was about 0.479 to 0.607). Individuals P18 and P20 had a considerably higher proportion of alleles from one cluster than the other (q_cluster $1=0.947$, q_cluster $2=$ 0.887, respectively, Additional file 1: Table S2). Lastly, one of the individuals with $B$. officinalis morphology
(M2) shows low admixture from the B. crispa cluster (q_cluster $2=0.110$, Additional file 1: Table S2).

\section{Discussion}

\section{Hybridization between B. officinalis and B. crispa on} Xishan Mountain

Both species investigated in this study have a variable phenotype with regards to many morphological characters. Therefore, one major objective of this preliminary study is to ascertain that the apparent morphological intermediacy has indeed resulted from hybridization, rather than extraordinary variability of one of the species in this area, because morphological intermediacy is not invariably associated with hybrids [21]. As can be seen from the haplotype network analysis (Fig. 2), the two species are considerably differentiated, with one of the loci showing as many as 28 substitutions between alleles found in the two species (Fig. 2a). This large differentiation between the species makes the genetic identification of hybrids less ambiguous, and therefore, the heterozygote state of all morphologically intermediate plants at most loci gives strong evidence that these individuals are indeed hybrids of $B$. crispa and B. officinalis. 
That these two species can successfully form hybrids is additionally supported by the pollination experiments. While B. crispa was not successful as a pollen donor for $B$. officinalis, viable seeds produced by $B$. crispa when receiving pollen from $B$. officinalis were as much as $24 \%$ of conspecific viable seed set (5.44 / 22.27, Table 2), indicating that sufficient hybrid seeds can be produced in the wild to allow the establishment of hybrids under suitable conditions. Furthermore, B. crispa produces an exceptionally low percentage of viable seeds from conspecific pollinations when compared to conspecific pollination in B. officinalis (34\% opposed to $91 \%$, Table 2). One explanation for the low seed production could be that the pollination experiments were carried out on transplanted plants, introduced to KBG, and that conditions at KBG are reflecting the ecological requirements of $B$. officinalis much better than those of B. crispa, hence $B$. crispa plants might have suffered substantial stress against the seed production. It would therefore have been desirable to perform the pollination experiments in the wild population at Xishan. However, this was not possible due to lack of permission from the local authorities, and the interference of the frequenting public. Another explanation could be the significantly different pollen viabilities of the two species (B. crispa $50 \%$ vs. B. officinalis $81 \%$ ). However, it would be expected that insufficient viable pollen would foremost affect the number of seeds produced, not the viability of produced seeds. As the number of successfully fertilized flowers (55\%, $43 \%$; Table 2) and seeds per fruit (both $~ 65$, Table 2) is nearly equal for both species, it seems unlikely that pollen viability had a significant effect.

\section{Hybrid zone composition and introgression}

Despite the relatively low number of fertile hybrid seeds, as compared to seeds resulting from conspecific pollination (5.44 vs 22.27, Table 2), hybrids are frequent in the investigated area. Most of these hybrids are most likely to be F1s as identified by the NewHybrids analysis, and supported by their heterozygous state, always one allele each from each species, for all investigated loci. Hybrid zones which comprise prevalently F1 individuals have been reported before [10, 22, 23], and it has been hypothesized that a high frequency of F1 individuals can under certain circumstances impede interspecific gene flow effectively. For example in Rhododendron and Encelia, F1 individuals effectively outcompete all other hybrid classes, thereby impeding backcrossing and thus introgression $[10,11]$. Under such circumstances the reproductive barrier seems to be mostly ecological as the parental species interbreed freely, and the F1 hybrids are highly fertile. The pollination experiments, however, point to a larger role of intrinsic incompatibilities, as opposed to ecological selection against hybrids, as the viable seed set for interspecific pollinations is lower than for intraspecific pollinations (Table 2). Furthermore, although most hybrids are F1s, some later generation hybrids were identified in the hybrid zone indicative of at least some successful backcrossing; two individuals of the morphologically intermediate individuals showed admixture with much higher contribution from one of the two species than would be expected for first generation hybrids (P18 and P20, Fig. 3). Additionally, several of the $B$. crispa individuals showed a low fraction of alleles derived from $B$. officinalis (Z6, Z13, Z15, Z17, Z22, Fig. 3). This relatively high number of $B$. officinalis alleles in a $B$. crispa background hints towards asymmetric introgression into this species. Due to factors such as phenology, gametopytic-sporophytic interactions during fertilization or organelle-nuclear gene interactions, asymmetric barriers in plants are quite common [24, 25], however, to elucidate which factors are most important for the present case requires further research.

Judging from the pollination experiments, a low number of backcrosses is expected due to the fact that the number of viable seeds produced for those crosses is relatively low (BC officinalis 1.11 and 2.45; BC crispa 1.54 and 0, Table 2). Additionally, pure B. officinalis and pure $B$. crispa individuals are more abundant in the population, making intraspecific pollinations, and interspecific pollinations between the parents, resulting in F1 offspring, more likely. Hence the occurrence of only few backcrosses can be expected, and their presence indicates that ecological selection is not strong enough to completely impede gene flow between the two species. Furthermore, from the individuals comprising the population at Xishan, one exhibiting pure $B$. officinalis morphology had a B. crispa allele at the gapC2 locus (M02), and four individuals with $B$. crispa morphology (Z06, Z09, Z13, and Z14) showed different levels of admixture (Fig. 3), suggesting occasional backcrossing, and thus the possibility of introgression. Interestingly the data from the pollination experiments suggests that theoretically, assuming conditions only taking the production of viable seeds into account, more backcrosses towards B. officinalis could be expected. Generally seed set was low when a hybrid individual was used as one parent, but crosses with $B$. officinalis produced some viable seeds in each direction, while crosses with $B$. crispa were only successful with the hybrid as pollen donor (Table 2), suggesting that more backcross seed involving $B$. officinalis should be produced. Due to the small sample size it is possible that this is an artifact, but it is also possible that certain other factors favor backcrossing to $B$. crispa in the wild. It is widely accepted that many types of pre- and post- zygotic barriers can act together to prevent hybridization and introgression [26-28]. Artificial pollination experiments only cover a small subset of these barriers. For instance temporally variable barriers 
such as flowering period, pollinator preference and seedling establishment of hybrids in nature are very difficult to assess, and might have lead to a higher occurrence of backcrosses towards B. crispa. However, at least during the seedling stage ecological selection is likely to affect successful establishment, and with the present data adaptively favored introgression, mostly benefiting individuals with B. crispa background can not yet be ruled out.

Because hybrids had never before been reported for this species pair, and additionally during extensive fieldwork throughout the distribution range of B. crispa, hybrids had never been observed, the reference population for B. crispa was sampled relatively close to Xishan Mountain, as we intended to avoid large allelic differences due to distance between sampled populations. The reference site, Yimen, was therefore also situated at the southern extreme of the distribution of B. crispa. The Structure analysis indicated that several $B$. crispa individuals in this population show admixture from $B$. officinalis (Z15, Z17, Z18, Z20-22; Fig. 3), giving evidence that hybridization is not restricted to Xishan Mountain, and that introgression also occurred in this population. Due to the distinctiveness of the B. crispa and B. officinalis alleles it is unlikely that this pattern could have been caused by ancient shared alleles, and a more in-depth search around the sample site revealed several morphologically intermediate individuals. As the population was assumed to be pure, examination during the first collection was not thorough, and likely the genetically admixed individuals would have been identifiable by means of morphology.

Some of the admixed individuals were morphologically not distinguishable from pure individuals of one or the other of the parental species. Therefore, a more comprehensive sampling approach will be needed in future studies to investigate potential past admixture in areas where the species distributions overlap. We are not aware of other publications demonstrating hybridization between the two species, and previous observations made during fieldwork, covering a wide range of the overlap of the two species [17] (Sun Weibang, Chen Gao personal observation), did not hint towards hybridization at other localities, hence the present data suggest a difference in reproductive isolation between the two species at the southern edge of the distribution of B. crispa as opposed to the rest of the distribution range. If $B$. crispa is advancing its range southwards, this might be expected, as according to theory introgression of local genes will often accompany a range expansion [29]. If some of the introgressed alleles are, however, adaptive remains unclear.

\section{Conclusions}

The occurrence of morphologically intermediate individuals between $B$. crispa and B. officinalis at Xishan
Mountain is unequivocally linked to hybridization. Morphologically intermediate individuals were almost all F1s, but some individuals which were classed as one of the parental species seem to be backcrosses, or show introgression. The two species can produce viable hybrid seed under controlled conditions, and backcrossing in both directions is theoretically possible. Later generation backcrosses and introgression were detected at both $B$. crispa sample sites, and the data suggests gene flow in both directions, as one individual identified as $B$. officinalis showed low amounts of admixture originating from the $B$. crispa cluster. Furthermore, at least at Xishan Mountain there is evidence that this introgression is mostly asymmetric, as a substantially higher proportion of $B$. officinalis alleles was detected in B. crispa than B. crispa alleles in $B$. officinalis.

\section{Methods}

\section{Plant sampling for molecular analysis}

Comprehensive field surveys involving $B$. crispa and $B$. officinalis have been performed in the last decade (Sun Weibang, personal observation). Although the two species are sympatric in some regions, only one putative hybrid zone has been identified on Xishan Mountain, Kunming, Yunnan, China (Fig. 4), where many individuals with intermediate morphology between $B$. crispa and B. officinalis were observed (Fig. 1). These individuals were hypothesized as natural hybrids, and mostly occurred along a main road in the scenic area of Xishan. B. officinalis individuals can be found throughout the area, and certainly more than 500 individuals can be found on Xishan; a population size estimate of B. crispa is more difficult, as the plants grow on sheer cliffs, but in the area more than 100 plants should be present. In this study, 24, 20 and 23 individuals of B. crispa, B. officinalis and their putative hybrid were collected respectively. All hybrids and some of the parents were collected at Xishan; additionally, further individuals of B. crispa and B. officinalis were collected at Yimen and Kunming Botanical Garden (KBG), respectively (Table 3). B. crispa and $B$. officinalis were identified according to the morphological descriptions in the Flora of China [12]. The eight individuals from Yimen, were collected without thorough checking for hybrid characters, as the population was assumed to be pure. Therefore it is possible that some of the later identified hybrids would have showed intermediate characters. Directly after collection, leaf material was transferred to zip-lock plastic bags containing silica gel.

\section{Measurements and data analysis of morphological traits}

Three leaves from ten healthy individuals for each of the three taxa were sampled from Xishan Mountain and then taken to KBG for morphological measurements. 


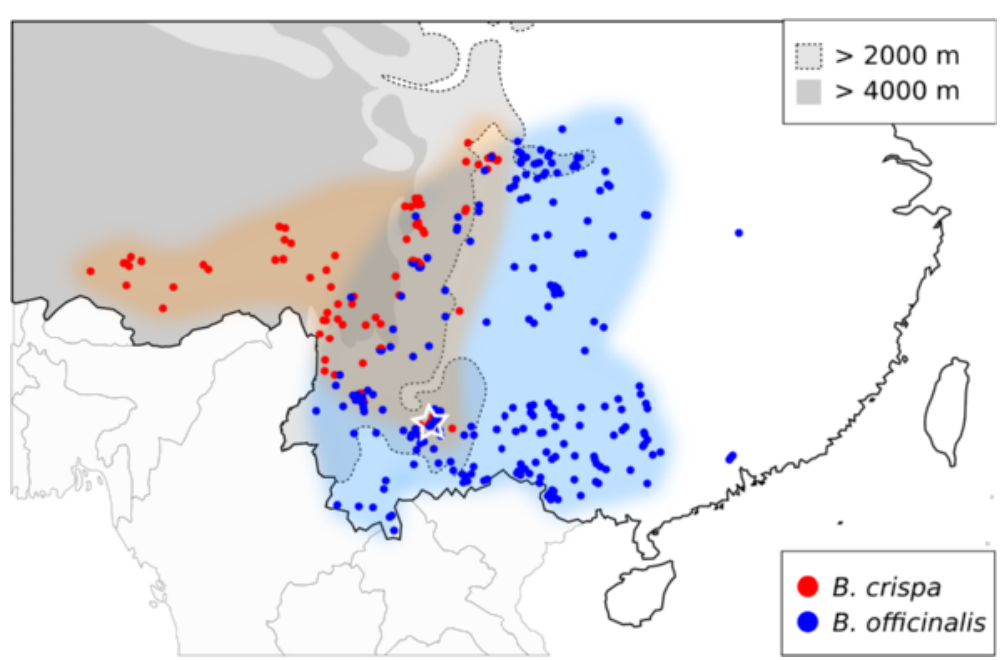

Fig. 4 Geographical distribution of B. officinalis (blue) and B. crispa (red) in China, based on locality information of 710 specimens (474 B. officinalis and 236 B. crispa); data for the specimens were obtained from the Chinese Virtual Herbarium (http://www.cvh.org.cn, accessed Aug 22, 2014), and all available specimens were included. B. crispa is predominantly found at altitudes $>2000 \mathrm{~m}$, while B. officinalis grows mostly at lower altitudes.

The location of the study site is indicated with a star

These samples were collected independently from the molecular samples and any overlap between them would be coincidental. Six leaf characters were assessed as follows: leaf indumentum, leaf shape, leaf base, leaf margin, leaf length ( $\mathrm{L}$, from the tip of the leaf to the position where the petiole joins the lamina) and leaf width (W, width at widest point). Additionally the flower color was noted for each of the individuals. Traits were analyzed with one-way ANOVA, where specie was treated as a fixed factor. When variances within each taxon were equal, as determined by a Levene test for homogeneity of variances, a standard $\mathrm{F}$ statistic was used to determine the significance of differences between means. When the data variances were different between groups the Welch statistic was employed. And significance of pairwise differences was assessed post hoc using a Tamhane test. All tests were performed as implemented in the SPSS package (SPSS 13.0 for Windows; SPSS, Chicago, Illinois, USA).

\section{Pollen viability test and hand pollination experiments}

Pollen stainability is the common methodology for detecting pollen viability. In the present study we use stainability of pollen with 2, 5-diphenyl tetrazolium bromide (MTT) as an indication of pollen viability [30]. MTT is a vital dye that detects the presence of dehydrogenase by the indication of purple color change for viable pollen grains [31], and the best condition for checking the viability of Buddleja using $0.1 \%$ MTT in $20 \%$ sugar solution had been detailed described by Gong [13]. Fresh pollen grains from 4 individuals of each parental species were collected from just-dehisced anthers during the blooming period, and stained with MTT to assess the viability. Then photographs were taken and the percentage of viable (stained) pollen was then calculated. Data was examined for normal distribution with a one sample Kolmogorov Smirnov test. For pairedcomparisons between treatments, independent-samples $t$ test was included. Data analysis was performed using SPSS 15.0 for Windows (SPSS, Chicago, IL, USA).

Hand pollination experiments were carried out in March 2013 in KBG where B. crispa and putative hybrids from Xishan Mountain were successfully introduced several years before. It would be desirable to conduct the pollination experiment in the study area, however it is impossible and not permitted to mark a label and bagging flowers for each sampling tree in such a scenic area with so many tourists each day. Three flowering plants of each taxon were selected to carry out the pollination experiments. For each of the conspecific pollinations the pollen of two of the plants in the same group was mixed to pollinate the third. For heterospecific

Table 3 Sampling details of the three Buddleja taxa in this study

\begin{tabular}{llll}
\hline \multirow{2}{*}{ Taxon } & \multicolumn{2}{l}{ Number of individuals sampled (Sample ID) } & Kunming Botanical Garden, Kunming \\
\cline { 2 - 4 } & Xishan, Kunming & Yimen, Yuxi & 8 (M13-M20) \\
\hline B. officinalis & 12 (M01-M12) & 0 & 0 \\
B. crispa & 14 (Z01-Z14) & 10 (Z15-Z24) & 0 \\
Putative hybrid & 23 (P01-P23) & 0 & 0 \\
\hline
\end{tabular}


pollinations the pollen of all three plants in one group was mixed to pollinate each of the six individuals not in the group. For each of the three possible cross-pollinations (one within the group, one from each of the other two groups), 8-15 flowers were randomly selected from each flowering plant. Emasculated flowers were handpollinated, and then bagged. In some cases pre-allocated flowers were accidentally damaged before the process was completed, resulting in 20 to 45 successfully pollinated flowers for each of the cross-pollinations. In May 2013, fruits were harvested, and seed numbers were counted.

Seed viability tests were carried out using an X-ray image system (MX-20-DC12, Faxitron, USA, [28]). It should be noted that seed numbers were counted after seed dispersal, as fruit ripeness is difficult to assess in Buddleja. Seeds of Buddleja taxa in this study were sometimes dispersed when fruits were still green.

\section{PCR amplification and sequencing of four low-copy genes and nrETS}

Total genomic DNA was extracted from silica-dried leaves using a modified CTAB method [32, 33]. The standard protocol was changed as follows: Dried leaves were ground to a fine powder in a Tissue Lyser (Qiagen), and no liquid nitrogen was used; PVP (Polyvinylpyrrolidone) was added to the CTAB extraction buffer. Sequences were obtained for four nuclear loci (gapC1, gapC2, fl1 and defA) and the external transcribed spacer of nuclear ribosomal DNA (nrETS). To design primers for these regions we first used eight pairs of universal primers for angiosperms. Of these primers only the primers for gapC worked for amplification in Buddleja [34]. These primers amplified two fragments of different length, which, based on sequence homology, turned out to be members of the gapC gene family. The two regions were therefore designated as gapC1 and gapC2, and two pairs of specific primers were designed for them. We then searched the GenBank for nuclear genes of the genus Buddleja and found sequences of three nuclear genes. Based on sequences of fl1 (Floricaula/Leafy-like protein 1, accession number DQ196438) and defA (a MADS box transcription factor of Buddleja davidii, accession number HQ853377), we designed primers for the two loci. The nrETS region was amplified using the universal primers ETS and 18S-IGS [35]. Sequences of all used primers are listed in Additional file 1: Table S3.

PCR was conducted using LA Taq DNA polymerase (Takara, Dalian, China) with the following conditions: initial denaturation at $94{ }^{\circ} \mathrm{C}$ for $4 \mathrm{~min}$, followed by $30 \mathrm{cy}$ cles of $94{ }^{\circ} \mathrm{C}$ for $40 \mathrm{~s}, 53{ }^{\circ} \mathrm{C}$ (nrETS, gapC1 and gapC2) or $52{ }^{\circ} \mathrm{C}$ (defA and fl1) for $45 \mathrm{~s}$, and $72{ }^{\circ} \mathrm{C}$ for $75 \mathrm{~s}$; finishing with a final extension at $72{ }^{\circ} \mathrm{C}$ for $10 \mathrm{~min}$. The PCR products were purified by running them on a $1.2 \%$ agarose gel, followed by extraction using a Pearl Gel Extraction Kit (Pearl Biotech, Guangzhou, China), and were then directly sequenced on an ABI 3730 DNA Analyzer using the BigDye Terminator Cycle Sequencing Ready Reaction Kit (Applied Biosystems, Foster City, California, USA). Intra-individual length polymorphism for the nuclear genes could cause failure of direct sequencing from the polymorphic sites. In addition, some individuals, mainly from the putative hybrid, had superimposed chromatograms at multiple sites of the nuclear genes, and the haplotypes could not be reliably inferred. Under these circumstances, cloning sequencing was used to phase the haplotypes. Ligations were conducted using the pMD18-T\&A cloning kit (Takara, Dalian, China). Eight positive clones for each individual were selected for sequencing.

\section{Sequence analyses}

Sequences of the four nuclear loci and nrETS regions were aligned and compared in SeqMan ${ }^{\mathrm{min}}$ (DNASTAR, Madison, Wisconsin, USA). As nrETS is generally believed to be homogenized by concerted evolution [36, 37], we treated nrETS, as a single locus, despite the presence of numerous copies in most plant genomes [37]. For B. crispa and B. officinalis, haplotypes were inferred as implemented by PHASE in DNASP5.0. Haplotype networks were constructed for each locus using Network 4.6.1.0 with the median-joining algorithm [38]. The program NewHybrids was used to assign each individual to a genotype category (parents, F1, F2, backcrosses) using the default settings [39]. This approach does not require that parental populations are sampled separately [39], assuming that only two generations of crossing have occurred. Using this program requires certain assumptions about the markers used: being unlinked, not subject to selection, and at linkage equilibrium in the parental species before hybridization [11]. We thus treated each haplotype as an allele, and conducted linkage disequilibrium test using the program Arlequin ver 3.5.1.3 [40]. Tajima's neutrality test was conducted for each locus in each parental species in DnaSP v5 [41]. We found no evidence for linkage disequilibrium between these loci in the parental species and for selection at each of these loci.

Genomic admixture proportions of all individuals were assessed using the program Structure version 2.3.1 [42]; the default settings were used, employing the admixture model with correlated allele frequencies. Run parameters were set to 100,000 iterations of MCMC, preceded by a burn-in of 100,000 . No prior knowledge of the species was included, and no popflags were set. To determine the most likely number of clusters $\mathrm{K}$, we calculated $\Delta \mathrm{K}$ by performing nine runs for each $K$ ranging from 1 to $10[20]$. 


\section{Additional files}

Additional file 1: Table S1. - The probabilities of each genetic clusters of NewHybrids analysis. Table S2 - The probabilities of each genetic clusters of Structure analysis. Table S3 - Sequences of primers used in this study.

Additional file 2: Figure S1. Value of $\Delta K$ from the Structure analyses.

\section{Competing interests}

The authors declare that they have no competing interests.

\section{Authors' contributions}

YPM, RCZ and WBS conceived and designed the project. GC and WBS conducted field surveys. WCG and RLL performed the experiments. RLL, YPM analysed and interpreted the data. RLL, RCZ, YPM drafted the manuscript. WBS and TM revised the manuscript. All authors read and approved the final manuscript.

\section{Acknowledgements}

This work was supported by grants from the National Natural Science Foundation of China (grant no. 31200247, 91231106, 30970192, U1302262) and the Yunnan Natural Science Foundation (grant no. 2012FB180).

\section{Author details}

${ }^{1}$ Kunming Botanical Garden; Key Laboratory for Plant Diversity and Biogeography of East Asia, Kunming Institute of Botany, Chinese Academy of Sciences, Kunming 650201, Yunnan, China. ${ }^{2}$ University of Chinese Academy of Sciences, Beijing 100049, China. ${ }^{3}$ College of Life Science and Technology, Honghe University, Mengzi 661199, Yunnan, China. ${ }^{4}$ State Key Laboratory of Biocontrol and Guangdong Provincial Key Laboratory of Plant Resources, Sun Yat-sen University, Guangzhou 510275, China.

Received: 4 March 2015 Accepted: 3 June 2015

Published online: 18 June 2015

\section{References}

1. Rieseberg LH, Gerber D. Hybridization in the Catalina Island mountain mahogany (Cercocarpus traskiae): RAOD evidence. Conserv Biol. 1995;9:199-203.

2. Arnold ML. Natural hybridization and evolution. Oxford: Oxford University Press; 1997.

3. Rieseberg LH. Hybrid origins of plant species. Annu Rev Ecol Evol S. 1997;28:359-89.

4. Rieseberg LH, Carney SE. Plant hybridization. New Phytol. 1998;140:599-624.

5. Soltis PS, Soltis DE. The role of hybridization in plant speciation. Annu Rev Plant Biol. 2009;60:561-88.

6. Coyne JA. Ernst Mayr and the origin of species. Evolution; 1994;48:19-30.

7. Levin DA. The origin, expansion, and demise of plant species. Oxford: Oxford University Press; 2000.

8. $\mathrm{Wu} \mathrm{Cl}$. The genic view of the process of speciation. J Evolution Biol. 2001;14:851-65.

9. Schluter D. Ecological causes of speciation. In: Endless forms: species and speciation. Oxford: Oxford University Press; 1998.

10. Milne Rl, Terzioglu S, Abbott RJ. A hybrid zone dominated by fertile F1s: maintenance of species barriers in Rhododendron. Mol Ecol. 2003;12:2719-29.

11. Milne Rl, Abbott RJ. Reproductive isolation among two interfertile Rhododendron species: low frequency of post-F1 hybrid genotypes in alpine hybrid zones. Mol Ecol. 2008;17:1108-21.

12. Li P, Leeuwenberg A. Loganiaceae. In: Flora of China. Beijing: Science Press; 1996.

13. Gong WC. Reproductive biology of the Buddleja (Scrophulariaceae) species in Asia. UCAS; 2014.

14. Sun WB, Kong FC, Lam WHM, Zhou Y, Li CR. Ornamental germplasm resources of genus Buddleja L. in Yunnan. Acta Horticulturae Sinica. 2001;29:81-3.

15. Norman E. Buddlejaceae. In: Flora Neotropica Monograph 81. New York: The New York Botanical Garden; 2000.

16. Chen G, Gong WC, Ge J, Dunn BL, Sun WB. Floral scents of typical Buddleja species with different pollination syndromes. Biochem Syst Ecol. 2012:44:173-8
17. Yue $L L$, Chen G, Sun WB, Sun H. Phylogeography of Buddleja crispa (Buddlejaceae) and its correlation with drainage system evolution in southwestern China. Ame J Bot. 2012;99:1726-35.

18. Chen XM, Chen FH, Zhou AF, Huang XZ, Tang LY, Wu D, et al. Vegetation history, climatic changes and Indian summer monsoon evolution during the Last Glaciation (36,400-13,400 calyr BP) documented by sediments from Xingyun Lake, Yunnan, China. Palaeogeogr Palaeocl. 2014;410:179-89.

19. Ni J, Cao XY, Jeltsch F, Herzschuh U. Biome distribution over the last 22,000 year in China. Palaeogeogr Palaeocl. 2014;409:33-47.

20. Evanno G, Regnaut S, Goudet J. Detecting the number of clusters of individuals using the software STRUCTURE: a simulation study. Mol Ecol. 2005; 14:2611-20.

21. Rieseberg L, Ellstrand N, Arnold M. What can molecular and morphological markers tell us about plant hybridization? Crit Rev Plant Sci. 1993;12:213-41.

22. Beatty GE, Philipp M, Provan J. Unidirectional hybridization at a species' range boundary: implications for habitat tracking. Divers Distrib. 2010;16:1-9.

23. Kameyama Y, Kudo G. Clarification of the genetic component of hybrids between Phyllodoce caerulea and Phyllodoce aleutica (Ericaceae) in Hokkaido, northern Japan. Plant Spec Biol. 2011;26:93-8.

24. Tiffin P, Olson S, Moyle LC. Asymmetrical crossing barriers in angiosperms. P Roy Soc B-Biol Sci. 2001;268:861-7.

25. Turelli M, Moyle LC. Asymmetric postmating isolation: Darwin's corollary to Haldane's rule. Genetics. 2007;176:1059-88.

26. Coyne JA, Orr HA. Speciation. Sunderland: Sinauer Associates; 2004.

27. Natalis LC, Wesselingh RA. Post-pollination barriers and their role in asymmetric hybridization in Rhinanthus (Orobanchaceae). Am J Bot. 2012;99:1847-56.

28. Ma Y, Xie W, Tian X, Sun W, Wu Z, Milne R. Unidirectional hybridization and reproductive barriers between two heterostylous primrose species in northwest Yunnan, China. Ann Bot. 2014;113:753-61.

29. Currat M, Ruedi M, Petit RJ, Excoffier L. The hidden side of invasions: massive introgression by local genes. Evolution. 2008;62:1908-20.

30. Dafni A, Firmage D. Pollen viability and longevity practical, ecological and evolutionary implications. Plant Syst Evol. 2000;222:113-32.

31. Zhang X, Gituru RW, Yang C, Guo Y. Exposure to water increased pollen longevity of pondweed (Potamogeton spp.) indicates different mechanisms ensuring pollination success of angiosperms in aquatic habitat. Evol Ecol. 2010;24:939-53.

32. Doyle JJ, Doyle J. A rapid DNA isolation procedure for small quantities of fresh leaf tissue. Phytochem Bull. 1987;19:11-5.

33. Doyle J. DNA protocols for plants-CTAB total DNA isolation. In: Molecular techniques in taxonomy. Berlin: Springer; 1991.

34. Strand AE, Leebens-Mack J, Milligan BG. Nuclear DNA-based markers for plant evolutionary biology. Mol Ecol. 1997;6:113-8.

35. Wright SD, Yong CG, Wichman SR, Dawson JW, Gardner RC. Stepping stones to Hawaii: a trans-equatorial dispersal pathway for Metrosideros (Myrtaceae) inferred from nrDNA (ITS+ ETS). J Biogeogr. 2001;28:769-74.

36. Arnheim N. Concerted evolution of multigene families. Sinauer: Sunderland; 1983.

37. Poczai $\mathrm{P}$, Hyvonen J. Nuclear ribosomal spacer regions in plant phylogenetics: problems and prospects. Mol Biol Rep. 2010;37:1897-912.

38. Bandelt $H J$, Forster $P$, Rohl A. Median-joining networks for inferring intraspecific phylogenies. Mol Biol Evol. 1999;16:37-48.

39. Anderson EC, Thompson EA. A model-based method for identifying species hybrids using multilocus genetic data. Genetics. 2002;160:1217-29.

40. Excoffier L, Lischer HE. Arlequin suite ver 3.5: a new series of programs to perform population genetics analyses under Linux and Windows. Mol Ecol Resour. 2010;10:564-7.

41. Librado P, Rozas J. DnaSP v5: a software for comprehensive analysis of DNA polymorphism data. Bioinformatics. 2009;25:1451-2.

42. Hubisz MJ, Falush D, Stephens M, Pritchard JK. Inferring weak population structure with the assistance of sample group information. Mol Ecol Resour. 2009;9:1322-32. 